\title{
Laboreal
}

Volume $10 \mathrm{~N}^{\circ} 2$ | 2014

Análise ergonómica do trabalho e formação - Parte II

\section{El análisis del trabajo treinta años después de Ombredane y Faverge}

A análise do trabalho trinta anos depois de Ombredane e Faverge

L'analyse du travail trente ans après Ombredane et Faverge

Work analysis thirty years after Ombredane and Faverge

\section{Guy Karnas y Pierre Salengros}

\section{(2) OpenEdition}

Journals

Edición electrónica

URL: http://journals.openedition.org/laboreal/5009

DOI: 10.4000/laboreal.5009

ISSN: 1646-5237

\section{Editor}

Universidade do Porto

Referencia electrónica

Guy Karnas y Pierre Salengros, «El análisis del trabajo treinta años después de Ombredane y

Faverge ", Laboreal [En línea], Volume 10 №2 | 2014, Publicado el 01 diciembre 2014, consultado el 24 septiembre 2020. URL : http://journals.openedition.org/laboreal/5009 ; DOI : https://doi.org/10.4000/ laboreal.5009

Este documento fue generado automáticamente el 24 septiembre 2020.

Laboreal está licenciado com uma Licença Creative Commons - Atribuição-NãoComercial 4.0 Internacional. 


\title{
El análisis del trabajo treinta años después de Ombredane y Faverge
}

\author{
A análise do trabalho trinta anos depois de Ombredane e Faverge \\ L'analyse du travail trente ans après Ombredane et Faverge \\ Work analysis thirty years after Ombredane and Faverge
}

Guy Karnas y Pierre Salengros

\section{REFERENCIA}

Karnas, G., \& Salengros, P (1986). L'analyse du travail trente ans après Ombredane et Faverge. In R. Patesson. L'Homme et l'écran. Aspects de l'ergonomie en informatique (p. 331-340). Colloque “ L'ergonomie en informatique », 1985, Nivelles. Bruxelles : Ed.de l'Université de Bruxelles.

\section{NOTA DEL EDITOR}

http://dx.doi.org/10.15667/laborealx0214ks

1 Así pues, este año se cumplen treinta años desde que Presses Universitaires de France publicara la obra de OMBREDANE y FAVERGE consagrada al análisis del trabajo que retomaba en lo esencial las conferencias impartidas en el Seminario de Análisis del Trabajo de la Université Libre de Bruxelles (U.L.B.) por Faverge en 1953 y 1954. En esta obra que demostraba sobre todo la pertinencia de la construcción de una metodología adaptada a cada actividad profesional, se encontraban todas las bases de una corriente que, en los países francófonos y en numerosos países del Este, iba a adoptar el nombre de ergonomía psicológica por oposición a los modelos restrictivos y estrictamente experimentalistas del human engineering de los EE.UU y que iba a reconciliar el análisis 
del hombre y el de su actividad, integrando la mayoría de los grandes capítulos de la psicología del personal, es decir :

- la selección, orientación y promoción profesional ;

- la formación profesional ;

- la cualificación del trabajo.

2 junto al desarrollo de esta.

3 Estas son las finalidades esenciales del análisis del trabajo que encuentran una dificultad principal y que De Montmollin destacaba en su conferencia inaugural : el análisis del trabajo es en efecto a la vez método y objeto; por tanto, solo se puede concebir refiriéndose a un objetivo con respecto al cual es "método".

4 Los problemas metodológicos planteados por el análisis del trabajo solo se pueden resolver recurriendo a otros métodos y todas las presentaciones de esta tarde tenderán a presentar los principales métodos utilizados con sus implicaciones en general y en el caso particular de la informática.

Desde el punto de vista de dichos métodos y manteniéndose lo más cercano posible a su texto, Faverge arremete con bastante violencia en el Análisis del Trabajo contra lo que denomina el "método ensayista", es decir, el análisis del trabajo en términos de aptitudes por descomposición en operaciones, o incluso exclusivamente por medio de encuestas a los ejecutivos.

6 En oposición a dichas prácticas, indica la necesidad de plantear claramente el problema estudiado y definir sin ambigüedad el objetivo del estudio ; ambas premisas determinan la elección de "criterios que permitirán sancionar el valor de la solución propuesta para el problema planteado".

7 La cuestión se convierte en definitiva en la cuestión de las relaciones, subyacentes al análisis del trabajo, entre el objetivo, el contenido y el modo (o incluso entre las finalidades, el objeto y la técnica).

8 En 1955, Faverge hace sobre todo hincapié en lo que respecta al contenido: la informatización, el lenguaje de las comunicaciones (se dedica más de un capítulo a este tema y toda la obra muestra la importancia para Ombredane y Faverge de la información, de los signos utilizados en el trabajo). Así, se desprende que una buena parte de la actividad del analista del trabajo se consagrará a la explicitación, comprensión de dichos signos, del paso de sistemas de signos a otros, etc.

9 Con "L'adaptation de la machine à l'homme" que inaugura en 1958 la colección "Le psychologue", Faverge establece las dos primeras acciones de la ergonomía, la gestual y la informacional.

Después de 1972, es decir, tras las investigaciones respaldadas primero por la CECA y posteriormente por la OBAP (Oficina belga para el crecimiento de la productividad), replantea el contenido informacional del trabajo con respecto a otros, indicando objetos o niveles de análisis que conservan hoy día toda su pertinencia y que hemos denominado los cuatro componentes de la ergonomía y que solo mencionaremos para aquellos que no estén todavía familiarizados con ellas.

- trabajar es adoptar posturas y realizar gestos; 
- trabajar es tratar información, es "comunicarse" con la materia, sea directamente, sea mediante intermediarios más o menos complejos, que van desde el indicador, el botón, el pedal, hasta el ordenador o el robot, pasando por el autómata ;

- trabajar consiste también en regular procesos complejos originados principalmente por las interacciones entre los elementos de un sistema del que somos nosotros mismos uno de los elementos que es -esto es evidente- también un sistema ;

- trabajar es finalmente poner en juego procesos de pensamiento que gestionen las actividades anteriores; es por tanto aplicar algoritmos, heurísticas, representaciones, estrategias. Este el ámbito de lo que se ha pasado a ser la ergonomía cognitiva.

11 Como indicó Faverge, los cuatro "análisis que resultan de la constatación de la existencia de los cuatros componentes del trabajo mencionados pueden realizarse y se complementan mutuamente, pero tienen importancias desiguales según la naturaleza del trabajo y los objetivos del estudio". Lo importante es destacar que estas cuatro componentes "aparecieron" de verdad históricamente en el orden en el que se han citado en función de cierta evolución resultante de modificaciones tecnológicas y ergonómicas.

En la actualidad, ¿dónde nos encontramos? Junto a las indicaciones de estos autores, quisiéramos insistir aquí brevemente en tres puntos en particular, siendo conscientes de que se trata de una elección entre tantas.

El primer punto tiene relación con el contexto en el que el análisis del trabajo debe tener lugar en la actualidad y quisiéramos destacar algunas características. Estas características han caminado de la mano aunque no es sencillo desenredar la madeja.

Puede distinguirse la toma de poder del hombre sobre su actividad, los movimientos de humanización del trabajo y el rápido desarrollo de las técnicas informáticas en el marco de una crisis económica con sus consecuencias en la dinámica organizacional de la empresa.

\section{$1^{\circ}$ ) La toma de poder del hombre sobre su actividad}

En una investigación ya antigua realizada con motivo de una primera automatización de un servicio de tipo bancario, nos llamó la atención el juego de manipulaciones recíprocas entre los empleados y la organización. El número de empleados que poner en línea diariamente depende del volumen de órdenes recibidas y dicho volumen varía particularmente de uno a otro día. La actividad de previsión parece ser el nudo de la actividad global y la fuente de los fenómenos de actitud de las personas en el trabajo ; la previsión no la realiza una célula de gestión, si no una sola persona que conoce perfectamente las potencialidades de las unidades de trabajo y los factores que pueden repercutir en la actividad.

El estudio de comportamiento de esta persona muestra a la vez un conocimiento preciso del número de documentos esperados (teniendo en cuenta los días de la semana, el mes, el periodo del año, los días de vacaciones, etc.) pero también una manipulación de dicha previsión de acuerdo con las normas que es posible aprehender : se observa una sobrevaluación sistemática de la previsión para los días de baja y media actividad y al contrario una infraevaluación para los días de mucha actividad.

17 Esta política deliberada conduce a una estabilización del número de órdenes en una zona media. 
trabajos informatizados o en curso de informatización es porque en este caso el hombre puede sin duda verdaderamente "jugar" contra la máquina para demostrar a la organización la aberración de su sustitución por esta y aporta en efecto otra cosa que la máquina no puede asumir, como se ha demostrado con numerosos ejemplos.

\section{$2^{\circ}$ ) Los movimientos de humanización del trabajo y sus corolarios} propuesto por Ombredane y Faverge, una evolución sociológica, política y económica
actuó como tal para desarrollar la corriente en ocasiones calificada de humanización del trabajo. Dicho movimiento, en concurrencia con el que tiene en cuenta las preocupaciones ergonómicas más funcionales, ha centrado aún más la atención en las condiciones de trabajo del hombre y por tanto en las exigencias del trabajo, su

Laboreal, Volume 10 N² | 2014 
adaptación al operador humano y, en definitiva, en la necesidad de un análisis del trabajo.

actividad y de relación que el sujeto muestra fuera de su actividad profesional ; así, los estilos de ocio de los vendedores son muy diferentes de los estilos de ocio de los empleados administrativos. Un directivo de una empresa siderúrgica se expresaba así : "los problemas sociales se deben en general al hecho de que hay un desfase demasiado grande de confort de vida, de modo de vida entre el medio de la fábrica y el medio fuera de fábrica. Creo que hay que intentar conciliar lo más posible la concepción del trabajo en fábrica y las condiciones laborales con lo que existe fuera de la fábrica para que haya el mínimo de conflictos posible".

30 Si esta reflexión expresa bruscamente una imagen de la necesidad de un equilibrio entre las dos vidas como condición de armonía y satisfacción, hay que comprender sobre todo que su anclaje recíproco brinda una nueva perspectiva sobre el problema de las representaciones del trabajo para el hombre, así como sobre la de los sistemas de valores que transmite; se pueden analizar varios conceptos derivados de lo anterior: en caso de concordancia entre las dos vidas, el concepto de ampliación horizontal o vertical de las tareas de enriquecimiento, de reestructuración del trabajo, interés por las experiencias industriales como los grupos semiautonómos o la participación voluntaria a una nueva definición de la organización del trabajo. En caso de rivalidad temporal entre vida laboral y vida fuera del trabajo, hay posibilidades de reinterpretación de los umbrales de declaración de accidentes o de los factores que influyen sobre el absentismo. En ambas circunstancias, se observa una nueva interpretación del trabajo a través de una tipología de estilos profesionales del hombre que constituye así una segunda idea que queremos desarrollar.

31 La noción de "estilo" presenta múltiples ventajas cuando se estudia la motivación del hombre en el trabajo : la noción ya antigua de necesidad hacía referencia sobre todo a una evaluación cuantitativa (la necesidad se satisface o no), la ventaja de una realización o de una expectativa era más positivo puesto que implicaba un proyecto personal, la noción de "objetivo" hacía a su vez referencia a la búsqueda de un resultado, por tanto, de un objetivo logrado o no ; el concepto de estilo introduce ahora la multiplicidad de objetivos o de expectativas; el objetivo o el éxito ya no es el criterio del hombre como tal, pero trabajadores diferentes pueden buscar y alcanzar objetivos distintos. La unidad ya no es aquí la del analista que aporta sus criterios y su manera de evaluar, si no que es la del trabajador en sí ; el objetivo se ve así a partir del hombre más bien que a partir de la organización. Definiendo el estilo de un hombre en su vida 
profesional como el conjunto organizado de sus comportamientos que reflejan su concepción personal de la vida en el trabajo, pudiendo por otro lado extenderse esta definición a la vida fuera del trabajo, nuestro laboratorio se ha concentrado en el análisis y definición de tipologías de estilos en marcos profesionales diferentes.

Ya se trate de obreros, monitores de formación profesional para adultos, de estudiantes o de ergónomos, se puede caracterizar maneras distintas y coherentes de comportarse en la actividad en cuestión; dicha coherencia se confirma en todo caso mediante el recurso a técnicas de formalización como el análisis tipológico y el análisis factorial.

Por tanto, el enfoque desde el que la ergonomía contempla el trabajo se invierte aquí con respecto a la concepción clásica : el sujeto habla por él pero esto solo puede realizarse realmente a costa de una modificación de métodos en la aproximación al problema : la entrevista y el cuestionamiento sustituyen a la observación y al análisis de la actividad. Se observa claramente que dichas técnicas acompañan el movimiento ya destacado de influencia del hombre sobre su trabajo, de un mayor respeto que se profesa a la interacción psicosocial entre analista y analizado; este desplazamiento metodológico puede dar lugar a nuevas dificultades en la interpretación de los resultados puesto que al contrario que las técnicas de investigación directa, estas están sujetas a manipulación.

\section{$3^{\circ}$ ) El desarrollo de las técnicas informáticas en un contexto de crisis económica.}

Las consecuencias de la política de informatización de las empresas comienzan a aparecer en el sector terciario, en particular el de los bancos y es posible hoy día medir algunas repercusiones quizá momentáneas : para los sujetos directa y continuamente enfrentados a la codificación de datos en los servicios centrales, sentimiento de esclavitud a la máquina, pérdida de grados de libertad en lo que respecta a los procedimientos, la ejecución y el tiempo de trabajo, pérdidas de empleo, sentimiento de pérdida de autonomía en la relación con la máquina ; para los empleados de agencias en contacto con el público, problemas particularmente cruciales de dependencia de la máquina, sentimiento de despersonalización en la relación con los clientes, etc.

El cuadro que el investigador obtiene de su observación de estos procedimientos es con mayor frecuencia opaco tal y como están las cosas; lo más grave es sin duda que los procedimientos ergonómicos que son hoy día objeto de estudio aunque ya estén teóricamente resueltos traten todavía con mayor frecuencia sobre una primera adaptación a la máquina, por ejemplo por un estudio de la luminosidad de los indicadores, por la forma o la disposición de los caracteres, por estudios de iluminación o de contraste.

Las investigaciones comienzan apenas a tratar sobre los aspectos psicosociológicos de la relación del hombre con la máquina, por ejemplo por la puesta a punto de programas más flexibles, más adaptados a los operadores en este sentido que estos podrían integrar en su proceso personal de análisis, su creatividad y su personalidad: en este sentido, hoy día se han propuesto intentos pero por motivos evidentes de productividad y de respeto de los procedimientos, no han salido todavía realmente de los laboratorios. 
Ilustremos este asunto con un ejemplo de estudio realizado en una institución de tipo bancario :

El puesto estudiado es un puesto de introducción de datos. El estudio tiene lugar cuando se va a realizar un cambio tecnológico importante. Los trabajadores tienen como cometido codificar los datos relativos a las operaciones bancarias transmitidas a la sede central. Los datos se registran en bandas magnéticas mediante un terminal. Pero cuando hasta entonces los datos se proporcionaban a las operadoras en forma de fichas de las que cogían un paquete en una pila durante la jornada, se propone hacer aparecer secuencialmente una imagen de las fichas en vídeo.

El análisis de trabajo se volcó esencialmente en la situación anterior. Permitió poner en evidencia una regulación del rendimiento en función de la cantidad de trabajo prevista para la jornada (la cantidad de documentos que tratar es muy variable de un día para otro y el tiempo de presencia en el trabajo es igualmente variable).

Un examen más en detalle de los datos recopilados por análisis tipológico muestra la existencia de subgrupos de operadoras. En efecto, mientras que algunas disminuyen su rendimiento cuando la jornada resulta larga, otras lo aumentan y otras incluso no lo modifican en ningún aspecto.

Resulta claramente que estas regulaciones tienen su origen en la posibilidad que tienen los operadores de estimar en cualquier momento el trabajo realizado y la tarea que queda por realizar. Analizando entonces las reticencias de las trabajadoras con respecto al cambio de su puesto de trabajo, el autor concluye que la posibilidad de estimar el trabajo que queda por hacer y en consecuencia de dominar su puesto, su actividad, es decir, de controlar la incertidumbre en la que uno se encuentra, es la base de muchas resistencias.

2 Segundo punto: en este marco en el que hombre busca y tiene la posibilidad de modificar sus actividad con respecto a lo prescrito, el análisis del trabajo debe distinguir el análisis de la tarea y el análisis de la actividad o incluso de las conductas operatorias.

Distinguiremos en el análisis el qué y el cómo. ¿Qué hay que hacer y cómo lo hacen los trabajadores?

El análisis de la tarea, descriptivo y diagnóstico, incluirá :

(1) la situación de la tarea en su contexto ;

(2) la descripción de la tarea (objetivos, principios de construcción del dispositivo, normas de funcionamiento, programa de funcionamiento)

(3) la descripción con respecto a las variables de las que se ha podido demostrar la influencia sobre el comportamiento del operador (como las limitaciones, los factores de no fiabilidad, etc.).

El análisis de la actividad distinguirá esencialmente por una parte los aspectos observables $\mathrm{y}$, por otra parte, los mecanismos que rigen la organización de estos últimos, es decir, la regulación de la actividad (aspectos perceptivos, tratamiento de la información incluido el análisis de errores y de su producción).

Esta distinción entre el análisis de tareas y análisis de la actividad, Leplat la sitúa en 1980 con respecto a otra distinción que ubica la actividad en el centro de una corriente cuyo origen son las condiciones que la determinan (las condiciones de trabajo) y el 
resultado son las consecuencias que la actividad genera para el trabajador y para el sistema.

En cuanto a las condiciones, definiremos de manera clásica las condiciones internas y las condiciones externas: es decir, por una parte las características del trabajador (antropométricas, estado orgánico, edad, sexo, cualificaciones, experiencia, formación, personalidad, etc.) y por otra parte, las características del entorno de trabajo (condiciones físicas, técnicas, organizacionales, socioeconómicas, etc.).

51 En este contexto, la actividad aparece según Leplat como "la respuesta del individuo al conjunto de estas condiciones. La actividad puede aplicarse por una parte al propio cuerpo o a objetos materiales y por otra pare a representaciones. En el primer caso, se hablará de actividad física o manual y dicha actividad será observable. En el segundo caso, se hablará de actividad representativa o mental (o incluso cognitiva o intelectual), la actividad inobservable deberá inferirse a partir de distintos indicios".

Tercer punto y conclusión del silogismo de los dos primeros puntos mencionados : el análisis de la tarea y el análisis de la actividad presentan una metodología específica.

En lo que respecta a la tarea, Sperandio (1984) propone los siguientes puntos que deberían incluirse necesariamente en el análisis :

- Delimitación del sistema hombre-máquina que se observa.

- Elaboración de un "croquis de conjunto" que definirá al operador máquina, los datos, las acciones.

- Descripción dinámica del funcionamiento del sistema.

- Identificación de las exigencias del trabajo.

- Localización de las eventuales disfunciones.

Para llegar a esto, se recurrirá a técnicas variadas como por ejemplo :

1. Los esquemas espaciales (modelos de organización espacial).

2. Los esquemas en términos de comunicación de la información.

3. Modelos de organización secuencial :

3.1 Diagrama de secuencias operacionales.

\subsection{Organigrama.}

1. Modelos de organización de las interacciones.

2. Modelos del tipo catálogo de funciones.

3. Catálogos de funciones humanas puestas en juego.

4. Identificación de las exigencias para el operador (como las Tablas LEST, SAVIEM, Método de perfiles de puestos RENAULT).

5. Taxonomía de las disfunciones.

6. Los esquemas de procesos.

7. Los esquemas de la organización hombre-máquina.

8. Los esquemas funcionales hombre-máquina.

En comparación, Faverge proponía en un capítulo "Como realizar un análisis del trabajo", tres modos de análisis cercanos al análisis de la actividad del operador, es decir :
1. El aprendizaje personal.
2. La observación del obrero durante su trabajo.
3. El estudio de los indicios del trabajo. 
a los que añadía algunas técnicas hoy día clásicas de la recopilación de información útiles para los métodos :

1.1. Los métodos gráficos de los organizadores y especialistas del estudio de los tiempos.

1.2. El muestreo del trabajo.

1.3 La técnica de los incidentes críticos de Flanagan.

2. La utilización de aparatos de registro y

3. La experimentación en laboratorio.

Las técnicas utilizadas hoy día constituyen variantes y prolongaciones de esta enumeración.

\section{BIBLIOGRAFÍA}

Faverge, J.M. \& Al (1958). L'adaptation de la machine à l'homme. Paris : Presses Universitaires de France.

Faverge, J.M. \& Al (1966). L'ergonomie des processus industriels. U.L.B. :Editions de l'Institut de Sociologie, Bruxelles.

Faverge, J.M. \& Al (1972). L'analyse du travail, In Traité de Psychologie Appliquée - tome 3, Presses Universitaires de France, París.

Faverge, J.M. \& Al (1970). L'organisation vivante. U.L.B. : Editions de l'Institut de Sociologie, Bruxelles.

Karnas, G. (1985). L'analyse du travail, ronéo, Bruxelles. [1]

\section{NOTAS}

1. Nota del Comité Editorial: este texto es anterior al siguiente: Karnas, G. (1987). L'analyse du travail. In C. Levy-Leboyer \& J.C. Sperandio (ed.), Traité de psychologie du travail (pp. 609-625). Paris: PUF.

\section{RESÚMENES}

El análisis del trabajo de Ombredane y Faverge había desarrollado en 1955 una metodología original que situaba la selección, formación y cualificación del trabajo junto a la organización estricta de este. 
Los autores explicitan tres ideas que han surgido desde entonces y que son todavía el fundamento de las investigaciones en esta materia : el análisis del trabajo debe distinguir entre el análisis de la tarea y el análisis de la actividad; ambos análisis presentan una metodología específica y cualquier análisis del trabajo debe asumir nuevos factores que actúan en interacción, es decir, la toma de poder del hombre sobre su actividad si no sobre su tarea, la influencia de la crisis económica y sus repercusiones y el desarrollo de las técnicas informáticas y derivadas.

\section{AUTORES}

\section{PIERRE SALENGROS}

Université Libre de Bruxelles, Laboratoire de Psychologie Industrielle et Commerciale, Avenue F. Roosevelt, 50, 1050 Bruxelles, Belgique 